J. Dairy Sci. 97:5497-5507

http://dx.doi.org/10.3168/jds.2014-8067

(C) American Dairy Science Association ${ }^{\circledR}, 2014$.

\title{
Prediction of pregnancy viability in bovine in vitro-produced embryos and recipient plasma with Fourier transform infrared spectroscopy
}

\author{
M. Muñoz, ${ }^{* 1}$ A. Uyar, $\$ \ddagger^{1}$ E. Correia, ${ }^{*}$ C. Díez, ${ }^{*}$ A. Fernandez-Gonzalez,§ J. N. Caamaño, ${ }^{*}$ D. Martínez-Bello,\# ${ }^{2}$ \\ B. Trigal, ${ }^{*}$ P. Humblot,II C. Ponsart,, C. Guyader-Joly, ${ }^{* *}$ S. Carrocera, ${ }^{*}$ D. Martin, ${ }^{*}$ B. Marquant Le Guienne, \\ E. Seli,† and E. Gomez ${ }^{* 3}$ \\ *Centro de Biotecnología Animal-Servicio Regional de Investigación y Desarrollo Agroalimentario (SERIDA), Camino de Rioseco 1225, \\ La Olla - Deva, 33394 Gijón, Asturias, Spain \\ †Department of Obstetrics, Gynecology, and Reproductive Sciences, Yale School of Medicine, 310 Cedar street, LSOG 304B, \\ New Haven, CT 06520 \\ ‡Department of Computer Engineering, Okan University, Tuzla Kampusu, 34959, Tuzla, Istanbul, Turkey \\ $\S$ Servicios Científico Técnicos (Oviedo), Universidad de Oviedo, 33006 Oviedo, Asturias, Spain \\ \#U.T.E. Bos-Lugar de Bos, Guísamo 15640 Bergondo, A Coruña, Spain \\ IISwedish University of Agricultural Sciences, PO Box 7054, SE75007 Uppsala, Sweden \\ TUnion Nationale des Cooperatives D' Elevage et Insemination Animale (UNCEIA), Department of Research and Development, 13 rue Jouet, \\ 94704 Maisons Alfort, France \\ ${ }^{* *}$ UNCEIA, Station Expérimentale, 484 Chemin Darefin, 38300 Chateauvillain, France
}

\section{ABSTRACT}

We analyzed embryo culture medium (CM) and recipient blood plasma using Fourier transform infrared (FTIR) metabolomics to predict pregnancy outcome. Individually cultured, in vitro-produced (IVP) blastocysts were transferred to recipients as fresh and vitrified-warmed. Spent CM and plasma samples were evaluated using FTIR. The discrimination capability of the classifiers was assessed for accuracy, sensitivity (pregnancy), specificity (nonpregnancy), and area under the receiver operator characteristic curve (AUC). Within all IVP fresh embryos (birth rate $=52 \%$ ), high AUC were obtained at birth, especially with expanded blastocysts (CM: $0.80 \pm 0.053$; plasma: $0.89 \pm 0.034$ ). The AUC of vitrified IVP embryos (birth rate $=31 \%$ ) were $0.607 \pm 0.038$ (CM, expanded blastocysts) and $0.672 \pm 0.023$ (plasma, all stages). Recipient plasma generally predicted pregnancy outcome better than did embryo CM. Embryos and recipients with improved pregnancy viability were identified, which could increase the economic benefit to the breeding industry. Key words: bovine, embryo, recipient, pregnancy

\section{INTRODUCTION}

Improvement of pregnancy rates upon embryo transfer $(\mathbf{E T})$ to recipients is a major objective in cattle

\footnotetext{
Received February 19, 2014.

Accepted May 27, 2014.

${ }^{1}$ These authors contributed equally to the study.

${ }^{2}$ Current address: Embriovet S.L. Rois 18 K 15165 Bergondo, A Coruña, Spain.

${ }^{3}$ Corresponding author: egomez@serida.org
}

farming, with significant financial implications. This aim has become even more relevant with the current increase in the use of embryos selected for their genetic merit and the need for high-fertility recipients (Humblot et al., 2010; Ponsart et al., 2013). However, currently, the selection of in vitro-produced (IVP) cow embryos for ET is based on embryo morphology and development stage, although neither is a good predictor of IVP embryo survival (Peterson and Lee, 2003). Morphological evaluation is a nonobjective method that leads to discrepant judgments among evaluators (Farin et al., 1995). Equivocal classification alters pregnancy rates, seemingly affecting IVP embryos more than in vivo-derived embryos (Farin et al., 1999). Furthermore, factors associated with in vitro culture cause embryonic mortality at different developmental periods (Peterson and Lee, 2003), with a sharper decrease in survival rates following cryopreservation of IVP embryos (Xu et al., 2006; Bruyère et al., 2012). Therefore, improving prediction of embryo viability would increase the efficiency of ET programs, particularly when IVP embryos are concerned.

Several in vitro techniques have been proposed to assess embryonic quality. Approaches include hatching rate analysis, survival to cryopreservation, cell counts of the inner cell mass and trophectoderm, measurement of apoptotic cells (Gómez et al., 2008, 2013), and analysis of incidence of chromosomal anomalies (Jakobsen et al., 2006). However, the above procedures have limited or no practical application for ET because the target embryo usually cannot be transferred following evaluation. In cattle, embryonic cell biopsy is rarely used to evaluate incidence of chromosomal abnormalities, although it is used to detect and quantify expression of certain genes 
associated with developmental competence (SalilewWondim et al., 2010; Ghanem et al., 2011). However, gene expression techniques are not extensively used in the field, and biopsy may compromise embryonic viability (Ponsart et al., 2013). As an alternative to invasive techniques, the culture medium (CM) that surrounds the embryo has been analyzed in a search for specific compounds associated with embryo viability. Single molecules measured in CM in correlation with embryo viability include glucose, lactate, and pyruvate (Gardner et al., 2001); oxygen consumption is an additional correlated factor (Lopes et al., 2007). Global analysis of CM includes amino acid analysis (Sturmey et al., 2010), proteome profiling (Katz-Jaffe et al., 2009), and ion profiling by electrospray ionization-mass spectrometry in multiple pregnancies (Cortezzi et al., 2013). Recently, a noninvasive combined measurement of developmental kinetics and morphology with oxygen consumption allowed reliable prediction of pregnancy rates from IVP cattle embryos (Sugimura et al., 2012).

Together with the particular limitations exposed, the above techniques are constrained by uncertain efficiency and may be technically difficult to perform, time consuming and expensive, and require qualified operators. Therefore, more objective and simple approaches are required to accurately predict embryonic viability in cattle.

In the ET field, less attention has traditionally been paid to recipient selection than to embryo selection. In practice, selection of recipients is based on assessment of corpus luteum (CL) function, by rectal palpation or ultrasonography, and progesterone measurement. Such selection procedures help increase pregnancy rates, but also often exclude too many intrinsically fertile animals (Hidalgo et al., 2004; Siqueira et al., 2009). Therefore, developing efficient and systematic methods for recipient selection is a challenging and pertinent novel objective for cattle ET technology. Metabolic analysis of serum or plasma provides a global profile of the metabolic status. Blood perfuses essentially all living cells in the body and it is thought to carry information on virtually every cell type. Metabolic changes affect nutrient transport from blood to oviductal and uterine fluids (Leese et al., 2008). Thus, some compounds of plasma could reflect the ability of a female to act as a high-quality embryo recipient.

Metabolomics profiling technology may allow determination of the metabolites associated with embryo viability and pregnancy outcome (Bromer and Seli, 2008). Nontargeted metabolomic profiling refers to quantitation of all the peaks in the spectrum without associating the peaks to chemical structure of certain compounds. One of the core approaches of nontargeted metabolomics is metabolic fingerprinting (MF), which considers the whole metabolic profile as a pattern that can be used for sample classification. In human in vitro fertilization (IVF), after analyzing CM by Raman and near-infrared spectroscopy (NIR) as spectroscopy approaches, the MF obtained predicted viability in oocytes and embryos (Seli et al., 2007; Scott et al., 2008). This shows that IVP embryos bearing a high reproductive potential modify their CM differently compared with embryos that do not lead to pregnancy, although the use of NIR did not lead to increased pregnancy rates in randomized prospective trials (Hardarson et al., 2012). Studies in human assisted reproductive technology have classically analyzed embryo viability. However, to our knowledge, the ability of a recipient to carry a pregnancy to term has not been considered so far under metabolomic approaches in any mammalian species.

Recently, the metabolome of spent embryo CM was analyzed by using nuclear magnetic resonance. The study revealed differences in CM metabolite ratios between pregnant and nonpregnant women diagnosed by urinary analysis of the $\beta$ subunit of human chorionic gonadotropin ( $\beta$ hCG; Wallace et al., 2014). By using another spectroscopy technique, Fourier transform infrared spectroscopy (FTIR), we recently developed a noninvasive system that successfully predicted embryonic sex after analysis of spent CM (Muñoz et al., 2014a). However, monitoring individual embryos requires single-embryo culture, an objective not yet refined with simple medium in cattle (Goovaerts et al., 2010), in which IVP embryos benefit developmentally from group culture.

In the present study, we verified the improved performance of a single culture step versus conventional group culture in vitro, and analyzed CM and plasma by FTIR metabolomics to predict pregnancy viability, from both from the embryonic and the recipient sides. Metabolomic profiles issued from IVP embryos following a 24-h single culture step were analyzed. Accomplishing these objectives may improve the efficiency of assisted reproductive technologies and genetic selection in cattle through better selection of embryos and recipients.

\section{MATERIALS AND METHODS}

All experimental procedures were carried out in accordance with the European Community Directive 86/609/EC and sanctioned by the Animal Research Ethics Committee of Servicio Regional de Investigación y Desarrollo Agroalimentario (SERIDA, Asturias, Spain; license 30/01/0). Ovaries were collected from cows slaughtered in a commercial abattoir (Matadero Central de Asturias, Junquera-Bobes, Spain) and transported to the SERIDA laboratory in $\mathrm{NaCl}$ solution $(9 \mathrm{mg} / \mathrm{mL})$ with antibiotics. All re- 


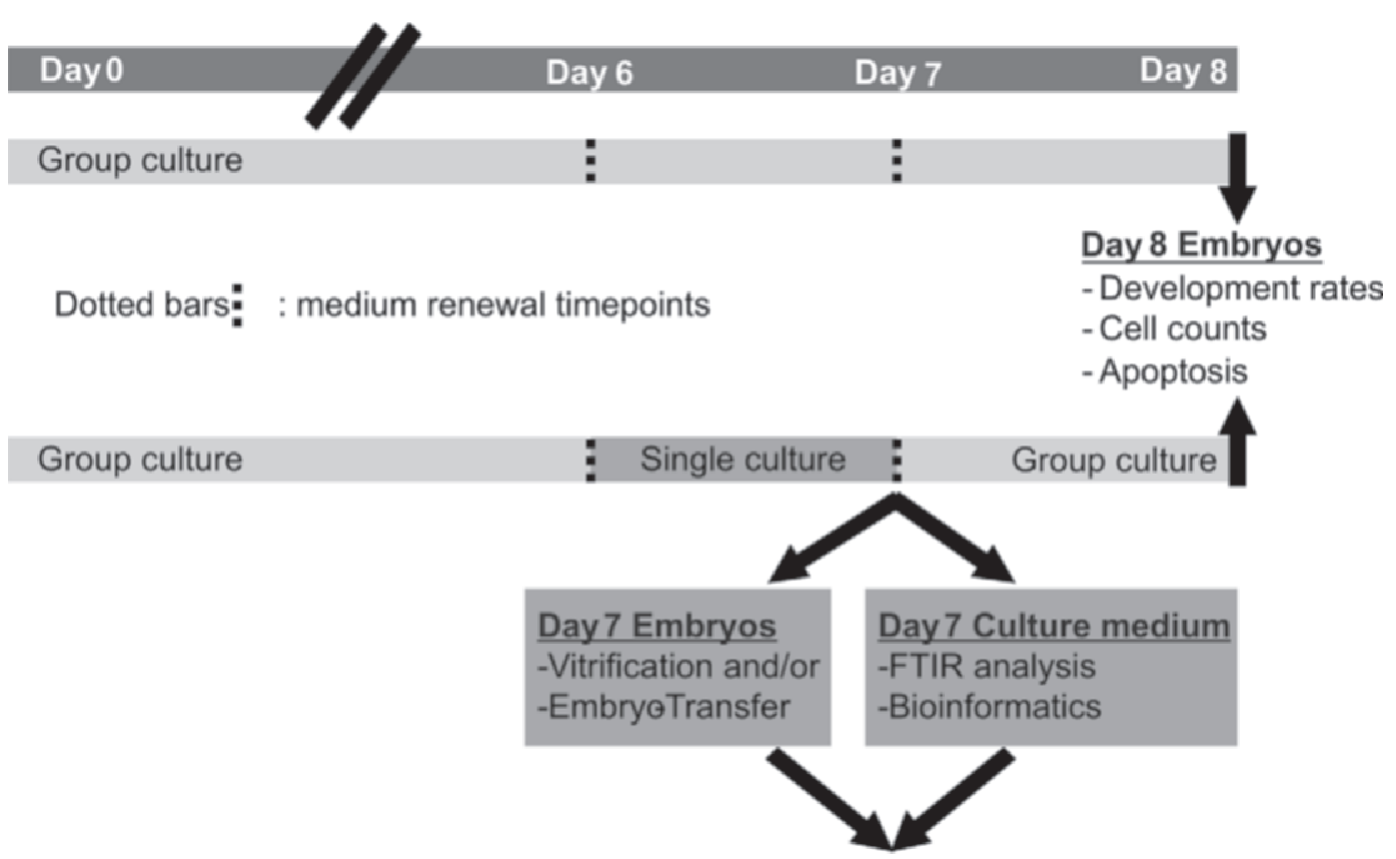

\section{VIABILITY PREDICTION}

Figure 1. Workflow of in vitro experiments conducted in this study leading to performance comparisons of single versus group culture and viability analysis in single culture medium of transferred embryos. FTIR $=$ Fourier transform infrared.

agents were purchased from Sigma (Madrid, Spain) unless otherwise stated.

\section{Optimization of Single Embryo Culture}

To analyze the metabolomic profile of individual embryos, it was necessary to establish and validate a $24-\mathrm{h}$ single embryo culture system and compare its efficiency to group culture. Ovarian follicles $(3-8 \mathrm{~mm}$ in diameter) were aspirated and cumulus-oocyte complexes recovered. After in vitro maturation for $24 \mathrm{~h}$, oocytes were in vitro fertilized (d 0) with unsorted semen from a single bull as described (Gómez et al., 2008). The CM was synthetic oviduct fluid (SOF) containing essential and nonessential amino acids, citrate, and myoinositol (SOFaaci) modified with $6 \mathrm{~g} / \mathrm{L}$ BSA. Presumptive zygotes were cultured in $50-\mu \mathrm{L}$ droplets under mineral oil, with 1 to $2 \mu \mathrm{L} /$ embryo, 25 to 35 embryos/drop, under $5 \% \mathrm{CO}_{2}$ and $5 \% \mathrm{O}_{2}$ in saturated humidity. Embryos were cultured in groups until $\mathrm{d} 6$. Under these conditions, embryos cleaved approximately at $85 \%$ on d 3 and produced $>40 \%$ morulae on d 6 . Subsequently, d-6 morulae (90\% approximately) and early blastocysts (10\% approximately) were picked up and individually transferred to either individual culture drops $(12 \mu \mathrm{L})$ for $24 \mathrm{~h}$ (d 6 to $\mathrm{d} 7$; single culture) or $50-\mu \mathrm{L}$ group culture drops (group culture with 25-35 embryos per drop). On d 7, embryos from the individual culture group were pooled and cultured as the group controls for an additional 24-h period. Media were renewed on d 6 and 7. Blastocysts and expansion and hatching rates were analyzed on $\mathrm{d} 7$ and 8 . Caspase- 3 staining and differential cell counts were determined in d-8 expanded and hatched embryos. Figure 1 shows a schematic representation of in vitro experimental procedures.

\section{Determination of Cell Counts and Caspase-3 Staining in Blastocysts}

Embryonic cells were differentially counted in the inner cell mass and trophectoderm of d-8 expanded and hatched blastocysts with a propidium iodide-bisbenzimide-based method as previously described (Thouas et al., 2001). Caspase-3 positive cells were counted in bovine d-8 expanded blastocysts following an immunostaining procedure previously described (Gómez et al., 2013). Briefly, fixed samples were permeabilized and blastomeric DNA was denatured before primary antibody incubation (\#9661, Cell Signaling Technology, Izasa, Barcelona, Spain). Caspase-3 positive cells were detected with the appropriate fluorescent secondary antibody and visualized by confocal fluorescence microscopy.

\section{Embryos Produced In Vitro for Embryo Transfer}

In vitro-produced embryos for transfer to recipients were fertilized with frozen unsorted ( 2 bulls) or sex- 
sorted sperm ( 5 bulls) as previously reported (Trigal et al., 2012). Embryos were cultured in SOFaaci and $6 \mathrm{~g} / \mathrm{L}$ BSA in individual culture from d 6 to $\mathrm{d}$ 7. On d 7, CM was recovered and stored frozen at $-80^{\circ} \mathrm{C}$ until FTIR analysis, and embryos were loaded in straws for ET as fresh or vitrified-warmed.

\section{Vitrification and Warming of Embryos}

Vitrification procedures have been described in detail previously (Trigal et al., 2012). Briefly, d-7 excellent and good expanded IVP blastocysts were vitrified in 2 steps using fiber plugs (Cryologic vitrification method, CVM; Equipos Médico-Biológicos, Barcelona, Spain). Vitrification solutions contained ethylene glycol, dimethyl sulfoxide, and sucrose as described (Vajta et al., 1998). Samples were vitrified by touching with the hook in a supercooled block placed in liquid nitrogen. For warming, the end of the fiber plug was immersed directly in 2 decreasing sucrose-containing solutions. Subsequently, embryos were washed twice and loaded in straws for ET.

\section{Culture Media and Embryo Recovery for Viability Analysis}

Spent CM $(10 \mu \mathrm{L})$ and blank controls (i.e., droplets incubated without embryos; up to 4 blank controls per batch of embryos cultured simultaneously) were collected on $\mathrm{d} 7$ and stored frozen at $-80^{\circ} \mathrm{C}$ until FTIR analysis. Embryos collected on d 7 were transferred, either fresh or after vitrification and warming, to estrussynchronized recipients (Muñoz et al., 2012).

\section{Estrus Synchronization of Recipients and Embryo Transfer}

Heifers were synchronized in estrus by using an intravaginal progestagen device (PRID Alpha, Ceva Salud Animal, Barcelona, Spain) for $10 \mathrm{~d}$ combined with a prostaglandin analog (Dynolytic, Pfizer, Madrid, Spain) injected $48 \mathrm{~h}$ before progestagen removal. On d 7 , single early expanding to expanded blastocysts (IVP-fresh or IVP-vitrified-warmed) were nonsurgically transferred to recipients in the cranial third of the uterine horn ipsilateral to CL under epidural anesthesia.

\section{Recipient Blood Sampling for Plasma Viability Analysis and Pregnancy Diagnosis}

Blood plasma samples from recipients were taken from coccygeal vein puncture and collected in EDTA evacuated tubes. Samples were taken at the time of standing estrus ( $\mathrm{d} 0)$ and on $\mathrm{d} 7(2-4 \mathrm{~h}$ before the ET time). Blood tubes were immediately refrigerated at $4^{\circ} \mathrm{C}$, and centrifuged at $2,000 \times g$ within 30 min after recovery. Supernatant plasma was aliquoted and stored at $-80^{\circ} \mathrm{C}$ until FTIR analysis.

Pregnancy was diagnosed by transrectal ultrasound scanning on d $60 \pm 2$ and birth date registered.

\section{FTIR Metabolomic Analysis}

Spent CM and blank samples were analyzed using a Golden Gate attenuated total reflectance (ATR) device (diamond crystal, Agilent Technologies, Madrid, Spain) mounted on a Varian 620-IR FTIR spectrophotometer running Varian Resolutions Pro software version 5.0.0.700 (Agilent Technologies). Five microliters of the sample was dropped on the ATR diamond and evaporated under a dry $\mathrm{N}_{2}$ flow until the FTIR spectrum was stable and different from that of water. The FTIR spectra (16 measurements per sample) were collected in a spectral range between 600 and $4,000 \mathrm{~cm}^{-1}$, at a speed of $5 \mathrm{kHz}$ and resolution of $4 \mathrm{~cm}^{-1}$. The relative standard deviation was $<3 \%$ at every wavelength in the range between 600 and $3,500 \mathrm{~cm}^{-1}$ (excluding the $\mathrm{CO}_{2}$ zone).

\section{Spectral Model Development}

The FTIR spectra obtained from CM and blood plasma were uploaded to the Matlab programming environment (R2011b; The MathWorks, Natick, MA) for data analysis and predictive model development. Two separate data sets were generated considering d-60 pregnancy and birth endpoints. In each data set, samples were labeled as 1 and -1 , associated with positive and negative outcomes, respectively. Binary (2-class) classification experiments were performed for model development.

The overall study population included spectra obtained from CM of IVP embryos transferred fresh (n $=27$ ) and IVP embryos transferred vitrified-warmed $(\mathrm{n}=42)$. Individual spectral profiles were normalized to the control medium to account for possible effects of variations in the culture conditions. Data corresponding to the $\mathrm{CO}_{2}$ frequency band $\left(2,285-2,400 \mathrm{~cm}^{-1}\right)$ were removed from the analysis. Each sample was then represented as a row vector of spectra data and the corresponding class label. Spectra from d 0 and d 7 plasma obtained from recipients to whom an embryo was transferred were also analyzed.

In a recent study, we performed a benchmarking experiment to assess the discrimination capability of a variety of classification algorithms on prediction of embryonic sex using CM spectra (Muñoz et al., 2014a). Among the classifiers tested, k-Nearest Neigh- 
bor (k-NN) provided the highest prediction accuracy. Therefore, we applied the k-NN method for viability prediction in this study. See Alpaydin (2010) for a more detailed explanation of $\mathrm{k}-\mathrm{NN}$ and other methods used in data analysis.

In the distance-based local k-NN model, the class label of a test sample is determined to be same as the most frequent class among its $\mathrm{k}$ neighborhood. The $\mathrm{k}-\mathrm{NN}$ method provides local solutions assuming that samples that are close together in the feature space will belong to the same class. The distances of each test sample to all training samples are calculated and sorted in ascending order. The majority of the class among shortest $\mathrm{k}$ distances is chosen as the class of the test sample. As the most general distance metric of k-NN algorithm, Euclidean distance was used in the experiments. The Euclidean distance $d(p, q)$ between the 2 points $p$ and $q$ in $N$ dimensional space is

$$
d(p, q)=\sqrt{\left[\sum_{i=1}^{N}\left(p_{i}-q_{i}\right)^{2}\right]} .
$$

Specifically, a weighted k-NN approach was applied where the contributions of neighbors to the class choice were weighted by the inverse of distances to the test sample. The study data set is a typical example of a high dimension low sample size (HDLSS) problem with 69 samples of CM, 137 samples from plasma, and 1,704 features obtained from spectroscopy analysis. We utilized principal component analysis (PCA) on the spectral data for dimensionality reduction.

\section{Training and Testing Strategy}

We applied 10-fold cross-validation training-testing strategy in the classification experiments. The entire data set was randomly divided into 10 bins. The predictive model was developed on 9 bins (training samples) and the performance of prediction was assessed on the remaining bin (test samples). To overcome sampling bias, the training-testing procedure was repeated 10 times, replacing the test samples with a bin from the training samples. The average results obtained from the repeated tests were presented. The discrimination capability of the classifiers was assessed in terms of accuracy, sensitivity (pregnant), sensitivity (nonpregnant), and area under the receiver operating characteristics curve (AUC; Uyar and Seli, 2012).

\section{Experimental Design}

In experiment 1 , embryos from 24-h single culture and group culture in vitro were compared. Embryo development was recorded on $\mathrm{d} 7$ and 8, and inner cell mass and trophectoderm cells counts and incidence of caspase- 3 cells were determined in d-8 blastocysts. In experiment 2 , we obtained the metabolomic profile of individually cultured embryos, upon FTIR analysis of frozen-thawed CM samples of IVP-fresh and IVP-vitrified-warmed embryos. Values were normalized against those of blank samples cultured without embryos. Predictive models compared pregnant with nonpregnant animals at d 60 and at birth within all categories of embryos analyzed. Specific studies on subsets of embryos were performed (i.e., fresh and vitrified IVP embryos). In experiment 3, we predicted pregnancy success from recipient plasma metabolomic analysis. For normalization purposes, in the absence of blank controls, we included $2 \mathrm{~d}$ of plasma sampling. Our aim was that subtraction of d 0 and d 7 plasma values could be an appropriate tool to normalize recipient data. Therefore, plasma spectral values were analyzed on $\mathrm{d} 0$ and $\mathrm{d} 7$, each being an independent prediction day, and normalized (i.e., d $7-\mathrm{d}$ 0).

\section{Statistics}

Data from embryo development, sex ratio, cell counts, and capase-3 staining were analyzed using the Proc GLM module of SAS/STAT (version 9.2; SAS Institute Inc., Cary, NC). The models included the following fixed effects: culture system, embryonic sex (where appropriate), and replicate. Least squares mean and their errors $( \pm$ SEM) were estimated for each level of fixed effects with a significant $F$-value. All spectral model development, data preprocessing steps, and postprediction statistical analyses were performed using Matlab (R2011b; The MathWorks). Classifier benchmarking tasks were conducted using Weka (Waikato Environment for Knowledge Analysis), an open-source data mining system (Witten and Frank, 2005). The significance of the differences between the predictive spectral models tested was assessed by comparing the associated AUC values using ANOVA or $t$-test when appropriate. An $\alpha$ error of $<0.05$ was considered significant for the comparisons.

\section{RESULTS}

\section{Assessment of 24-h Single Embryo Culture System}

Blastocyst development and expansion rates on $\mathrm{d} 8$ did not differ between single and group cultures (Table 1). However, embryos that were individually cultured during a 24-h step showed d-8 hatching rates almost double those of group-cultured embryos $(16.1 \pm 1.2$ vs. $8.5 \pm 1.2$, respectively; $P=0.018$ ). In addition, 


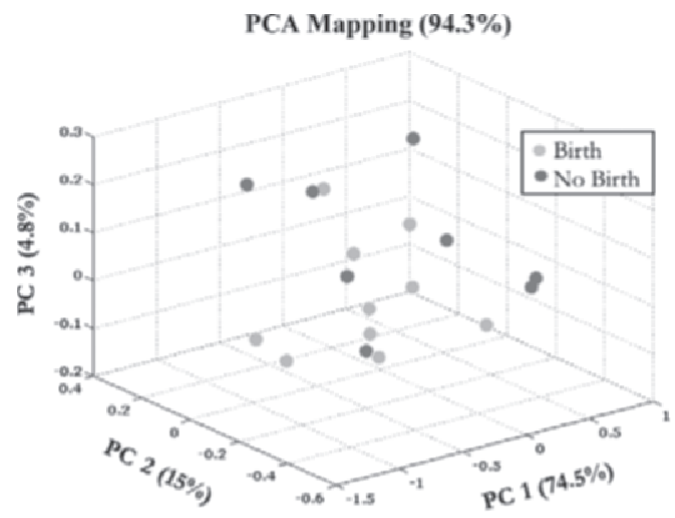

PCA Mapping (97.5\%)

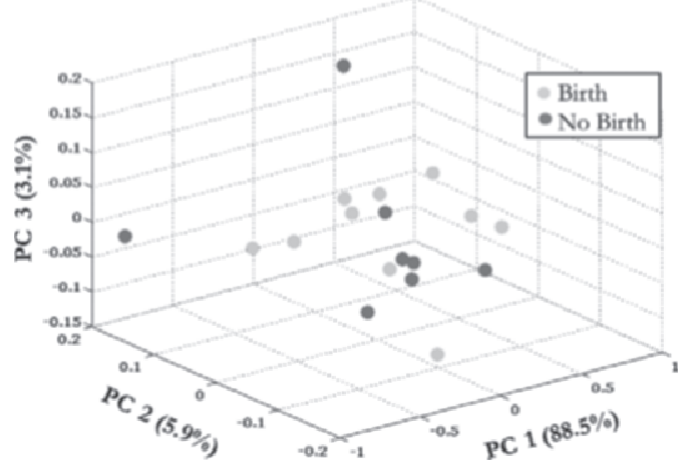

Figure 2. (A) Principal component analysis (PCA) of (A) culture media spectra obtained from fresh in vitro expanded blastocysts; and (B) d $7-\mathrm{d} 0$ plasma spectra obtained from recipients of fresh in vitro expanded blastocyst. The percentages of variability represented by the first 3 principal components are displayed on PC 1,2 , and 3 on the $\mathrm{x}-$, y-, and z-axes, respectively.

information on the likelihood of pregnancy and birth. The MF of CM seems to be influenced by treatments that an embryo underwent (i.e., cryopreservation) and ultimately by embryonic stage. In addition, recipients of fresh and vitrified-warmed embryos that became pregnant exhibited distinct plasma MF. However, the use of different bulls did not seem to affect the efficiency of viability predictions. Interestingly, information from recipients (plasma) was generally more predictive of pregnancy success than that from embryo CM.

In most laboratories, bovine IVP embryos are cultured in groups to improve developmental outcomes. However, single oocyte and embryo culture have unquestionable advantages. Efficient single culture of bovine embryos would allow treatment of cow-donors with low oocyte rates and using the bovine as a model for the human embryo culture. Unfortunately, developmental competence, embryo quality, and cryotolerance are affected by single culture, with only a few authors reporting similar or improved blastocyst development rates in single culture compared with group culture (Goovaerts

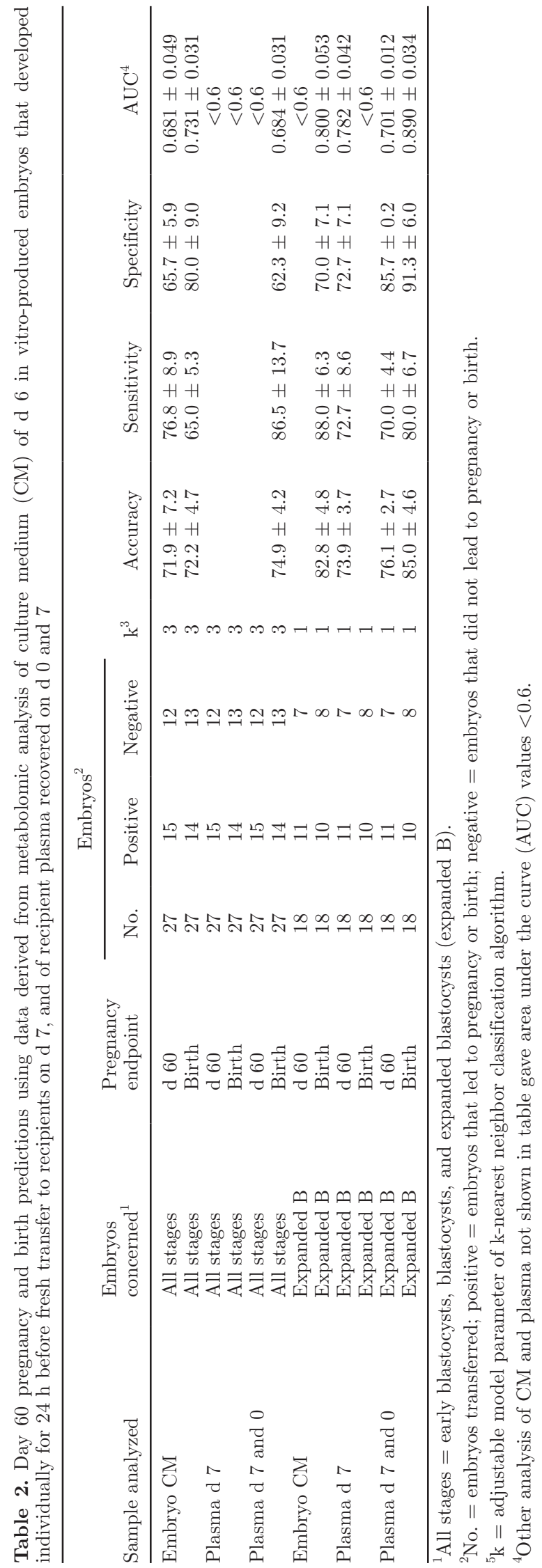

Journal of Dairy Science Vol. 97 No. 9, 2014 
et al., 2010). In our study, a simple, individual 24-h culture step, improved hatching at levels almost twice that of embryos cultured in groups, without triggering changes in zona-enclosed blastocyst development, cell counts, or incidence of apoptosis. The good performance of this single culture system was also revealed by fresh IVP embryos, which showed pregnancy and birth rates comparable to those of fresh in vivo embryos that were cultured for $24 \mathrm{~h}$ in another study (Muñoz et al., 2014b). It is noteworthy that IVP embryos usually show lower pregnancy and birth rates than their in vivo counterparts upon ET (Farin et al., 1999; Siqueira et al., 2009).

Culture medium from IVP fresh embryos was more predictive of birth than of d-60 pregnancy rates. This suggests that miscarriages in IVP embryos might be predicted in culture; that is, an embryo may show high viability at $\mathrm{d} 60$ but lower viability at birth. The transfer of IVP embryos compared with in vivo-derived embryos usually results in more abortions and more congenital abnormalities in the offspring (Farin et al., 2006). Sugimura and coworkers (2012) showed that a combination of techniques to select bovine blastocysts may predict the success or failure of pregnancy and neonatal health better than individual factors. The MF represents the effects of multiple factors in a single assay, and metabolomic changes marked in the CM may be affected by the different epigenetics of IVP embryos, which might underlie late embryonic losses (Young et al., 2001). Postnatal abnormality and certain pathologies could be predictable from the early periconception (Sinclair et al., 2007). Therefore, the CM of IVP embryos could be informative of not only late pregnancy losses but also postnatal health.

Classification by embryonic stage improved viability predictions of fresh and vitrified IVP embryos with CM and plasma. Within IVP embryos, expanded blastocysts show superior pregnancy rates (Hasler, 2000; Block et al., 2009) and improved survival to vitrification (Xu et al., 2006; Block et al., 2009). Expanded blastocysts can be easily distinguished from early embryonic stages by their increased diameter and thinner zona pellucida. In contrast, discrimination between early blastocysts and blastocysts can sometimes be difficult for the operator. Therefore, assessing these 2 stages in a single pool is recommended. Ultimately, a combination of assessment criteria that includes morphometry and morphology, embryo treatments, and metabolomics and bioinformatics analysis may yield the best predictive assessments of embryo viability. The expanded blastocyst was the most advanced stage analyzed in our work. It is likely that the CM collected at the end of expansion would reflect more molecular traces than $\mathrm{CM}$ at earlier developmental stages, leading to a better predictive profile 
with expanded blastocysts. However, the existence of a better predictive profile in recipient plasma (i.e., not influenced by the embryo) transferred with expanded blastocysts suggests that other factors may exist in the adaptation of recipients to expanded blastocysts. Thus, fewer requirements of synchronization of recipients with expanded blastocysts (Kubisch et al., 2004) could explain such an improvement in the predictive profile. In vitro-produced embryos and recipients are usually synchronized with an estimated tolerance of $\pm 12 \mathrm{~h}$ (Kubisch et al., 2004) to $-24 \mathrm{~h}$ (Reichenbach et al., 1992). We restricted embryo transfers to recipients showing estrus on $\mathrm{d} 7 \pm 12 \mathrm{~h}$, and synchronization did not represent a variable in our study.

Vitrification introduced a further variability step that could affect pregnancy predictions; thus, the expected predictability could be lower for vitrified embryos than for fresh embryos. However, predictive profiles observed with vitrified embryos were close to $(\mathrm{CM})$ or reaching (plasma) twice the overall pregnancy rates (i.e., 33\%) obtained with such embryos. The low survival to cryopreservation of IVP embryos has been related to an excess of lipid contained in embryonic cells (Sudano et al., 2012), and the chemical composition of such lipids could affect survival following cryopreservation (Sudano et al., 2012). Differences in lipid composition may indicate different mitochondrial or peroxisomal metabolism, which could impose a distinct embryo metabolomic profile in the CM between pregnant vitrified and fresh embryos.

Mitochondria are the major source of oxidative stress, which underlies the toxic effects of cryopreservation leading to decreased embryo survival (Martino et al., 2013). Early embryos induce in the cow uterus a developmentally improved environment with increased concentrations of antioxidant proteins in the uterine fluid (Muñoz et al., 2012). This recipient response could be more critical in vitrified-warmed embryos than in fresh embryos, provided that embryonic cell defense could be compromised by cryopreservation, which might explain differences in MF plasma profiles between recipients of fresh and vitrified embryos that became pregnant. Interestingly, the plasma recipient was generally more predictive of viability than the CM profile. In particular, normalized values of plasma better predicted birth rates than d-60 pregnancy. Ultimately, 2 sampling days might not be necessary if single plasma samples, taken on either d 0 or $\mathrm{d} 7$, predict better.

The ability of a recipient to maintain pregnancy until birth is thought to be a source of variation greater than the ability of the embryo to survive to term (McMillan, 1996; McMillan and Donnison, 1999). In addition, variation in recipient quality is not an important contribution to fetal loss from d 60 to term (McMillan, 1996).
Effective identification of the higher recipient variability by FTIR could explain the superior predictive ability of the animals compared with the embryo. The McMillan model predicts the existence of intrinsically superior recipients (McMillan and Donnison, 1999). Superior recipient heifers may show changes in endometrial expression of genes and proteins (Salilew-Wondim et al., 2010) affecting major metabolic pathways and immune response. Some ET practitioners are becoming aware of this and retain recipients that successfully delivered calves after ET for future transfers.

Calculating predictions as a function of the treatment of embryos transferred (i.e., data from fresh or vitrified) allowed us to obtain reliable results from $\mathrm{CM}$ and plasma, as overall calculations (i.e., data from fresh and vitrified) were nonpredictive. Furthermore, the plasma metabolomic profile of pregnant recipients from fresh and vitrified IVP embryos differed within the same herd. Collectively, these results indicate that recipients differ in their pregnancy ability depending on whether the embryo carried was fresh or had been cryopreserved. To our knowledge, the association of embryos with specific recipients on the basis of their metabolomic profiles has been not yet studied, and it might help optimize the use of recipients, including the rescue of females that are discarded for ET using conventional selection. In addition, as recipients and embryos can enter into an early dialog in which immunological concerns are prominent (Muñoz et al., 2012; Gómez et al., 2013), compatibility between specific embryos (CM) and recipients (plasma) may exist, a relationship worth investigation. Such an approach will be accomplished shortly in a prospective, blind study.

Recipient and embryo selection by FTIR-metabolomics, coupled with morphology analysis of embryonic stages, can lead to increased pregnancy rates upon transfer of fresh and vitrified-warmed IVP embryos. The ability to carry a pregnancy with IVP fresh or vitrified embryos may necessitate selection of specific recipients. Recipient selection by plasma analysis seems to be more effective than embryo selection by $\mathrm{CM}$ analysis, suggesting that FTIR could be also used to select recipients on field in conventional multiple ovulation ET programs without embryo culture (Muñoz et al., 2014b).

\section{CONCLUSIONS}

Fourier transform infrared analysis of CM provides a noninvasive, fast (10 min per sample processing and analysis), easy to handle, and inexpensive (average consumables $<€ 4$ per sample) method that requires minimal sample pretreatment. The method is compatible with the highest sanitary standards and guidelines for 
international exchanges of embryos. Using embryos and recipients with improved viability indices will significantly increase pregnancy rates and economic benefit in the cattle breeding industry. Effective pregnancy viability predictions will depend on the availability of larger databases produced under different laboratory conditions and herds.

\section{ACKNOWLEDGMENTS}

The authors thank F. Goyache [Servicio Regional de Investigación y Desarrollo Agroalimentario (SERIDA), Asturias, Spain] for valuable scientific comments; I. Fernández (SERIDA) for his assistance in preparing the art work; and Cooperativa de Agricultores de Gijón, Veterinary Service (Gijón, Spain). Project support was provided by the Spanish Ministry of Science and Innovation (MICINN, projects AGL2012-37772, AGL200910059 and RTA2011-00090). M. Muñoz, E. Correia, and B. Trigal are supported by MICINN-RYC08-03454, MEC-FPU-AP2009-5265, and Cajastur, respectively. E. Seli is supported by Award R01HD059909 from the National Institutes of Health (NIH; Bethesda, MD). The authors are members of the COST Action FA1201 Epiconcept: Epigenetics and Periconception environment.

\section{REFERENCES}

Alpaydin, E. 2010. Introduction to Machine Learning. MIT Press, Cambridge, MA.

Block, J., L. Bonilla, and P. J. Hansen. 2009. Effect of addition of hyaluronan to embryo culture medium on survival of bovine embryos in vitro following vitrification and establishment of pregnancy after transfer to recipients. Theriogenology 71:1063-1071.

Bromer, J. G., and E. Seli. 2008. Assessment of embryo viability in assisted reproductive technology: Shortcomings of current approaches and the emerging role of metabolomics. Curr. Opin. Obstet. Gynecol. 20:234-241.

Bruyère, P., A. Baudot, C. Guyader-Joly, P. Guérin, G. Louis, and S. Buff. 2012. Improved cryopreservation of in vitro-produced bovine embryos using a chemically defined freezing medium. Theriogenology 78:1294-1302.

Cortezzi, S. S., E. C. Cabral, M. G. Trevisan, C. R. Ferreira, A. S. Setti, D. P. Braga, R. D. Figueira, A. Iaconelli, M. N. Eberlin, and E. Borges. 2013. Prediction of embryo implantation potential by mass spectrometry fingerprinting of the culture medium. Reproduction 145:453-462.

Farin, P. W., J. H. Britt, D. W. Shaw, and B. D. Slenning. 1995. Agreement among evaluators of bovine embryos produced in vivo or in vitro. Theriogenology 44:339-349.

Farin, P. W., J. A. Piedrahita, and C. E. Farin. 2006. Errors in development of fetuses and placentas from in vitro-produced bovine embryos. Theriogenology 65:178-191.

Farin, P. W., B. D. Slenning, and J. H. Britt. 1999. Estimates of pregnancy outcomes based on selection of bovine embryos produced in vivo or in vitro. Theriogenology 52:659-670.

Gardner, D. K., M. Lane, J. Stevens, and W. B. Schoolcraft. 2001. Noninvasive assessment of human embryo nutrient consumption as a measure of developmental potential . Fertil. Steril. 76:1175-1180.

Ghanem, N., D. Salilew-Wondim, A. Gad, D. Tesfaye, C. Phatsara, E. Tholen, C. Looft, K. Schellander, and M. Hoelker. 2011. Bovine blastocysts with developmental competence to term share similar expression of developmentally important genes although derived from different culture environments. Reproduction 142:551-564.

Gómez, E., J. N. Caamaño, F. J. Corrales, C. Díez, A. Correia-Álvarez, D. Martín, B. Trigal, S. Carrocera, M. I. Mora, J. PelloPalma, J. F. Moreno, and M. Muñoz. 2013. Embryonic sex induces differential expression of proteins in bovine uterine fluid. J. Proteome Res. 12:1199-1210.

Gómez, E., A. Rodríguez, M. Muñoz, J. N. Caamaño, C. O. Hidalgo, E. Morán, N. Facal, and C. Díez. 2008. Serum free embryo culture medium improves in vitro survival of bovine blastocysts to vitrification. Theriogenology 69:1013-1021.

Goovaerts, I. G., J. L. Leroy, E. P. Jorssen, and P. E. Bols. 2010. Noninvasive bovine oocyte quality assessment: Possibilities of a single oocyte culture. Theriogenology 74:1509-1520.

Hardarson, T., A. Ahlström, L. Rogberg, L. Botros, T. Hillensjö, G. Westlander, D. Sakkas, and M. Wikland. 2012. Non-invasive metabolomic profiling of day 2 and 5 embryo culture medium: A prospective randomized trial. Hum. Reprod. 27:89-96.

Hasler, J. F. 2000. In vitro production of cattle embryos: Problems with pregnancies and parturition. Hum. Reprod. 15(Suppl. 5):4758.

Hidalgo, C. O., E. Gomez, L. Prieto, P. Duque, F. Goyache, L. Fernandez, I. Fernandez, N. Facal, and C. Diez. 2004. Pregnancy rates and metabolic profiles in cattle treated with propylene glycol prior to embryo transfer. Theriogenology 62:664-676.

Humblot, P., D. Le Bourhis, S. Fritz, J. J. Colleau, C. Gonzalez, C. Guyader Joly, A. Malafosse, Y. Heyman, Y. Amigues, M. Tissier, and C. Ponsart. 2010. Reproductive technologies and genomic selection in cattle. Vet. Med. Int. 2010:192787.

Jakobsen, A. S., P. D. Thomsen, and B. Avery. 2006. Few polyploid blastomeres in morphologically superior bovine embryos produced in vitro. Theriogenology 65:870-881.

Katz-Jaffe, M. G., S. McReynolds, D. K. Gardner, and W. B. Schoolcraft. 2009. The role of proteomics in defining the human embryonic secretome. Mol. Hum. Reprod. 15:271-277.

Kubisch, H. M., S. Sirisathien, P. Bosch, H. J. Hernandez-Fonseca, G. Clements, J. R. Liukkonen, and B. G. Brackett. 2004. Effects of developmental stage, embryonic interferon-tau secretion and recipient synchrony on pregnancy rate after transfer of in vitro produced bovine blastocysts. Reprod. Domest. Anim. 39:120-124.

Leese, H. J., S. A. Hugentobler, S. M. Gray, D. G. Morris, R. G. Sturmey, S. L. Whitear, and J. M. Sreenan. 2008. Female reproductive tract fluids: Composition, mechanism of formation and potential role in the developmental origins of health and disease. Reprod. Fertil. Dev. 20:1-8.

Lopes, A. S., S. E. Madsen, N. B. Ramsing, P. Løvendahl, T. Greve, and H. Callesen. 2007. Investigation of respiration of individual bovine embryos produced in vivo and in vitro and correlation with viability following transfer. Hum. Reprod. 22:558-566.

Martino, N. A., M. E. Dell'aquila, R. A. Cardone, B. Somoskoi, G. M. Lacalandra, and S. Cseh. 2013. Vitrification preserves chromatin integrity, bioenergy potential and oxidative parameters in mouse embryos. Reprod. Biol. Endocrinol. 11:27.

McMillan, W. H. 1996. Potential survival rates to term for transferred in vitro and in vivo derived embryos. Theriogenology 45:233. (Abstr.)

McMillan, W. H., and M. J. Donnison. 1999. Understanding maternal contributions to fertility in recipient cattle: Development of herds with contrasting pregnancy rates. Anim. Reprod. Sci. 57:127-140.

Muñoz, M., F. J. Corrales, J. N. Caamaño, C. Díez, B. Trigal, M. I. Mora, D. Martín, S. Carrocera, and E. Gómez. 2012. Proteome of the early embryo-maternal dialogue in the cattle uterus. J. Proteome Res. 11:751-766.

Muñoz, M., A. Uyar, E. Correia, C. Díez, A. Fernandez-Gonzalez, J. N. Caamaño, B. Trigal, S. Carrocera, E. Seli, and E. Gomez. 2014a. Non-invasive assessment of embryonic sex in cattle by metabolic fingerprinting of in vitro culture medium. Metabolomics 10:443-451.

Muñoz, M., A. Uyar, E. Correia, C. Ponsart, C. Guyader-Joly, D. Martínez-Bello, B. Marquant-Le Guienne, A. Fernandez-Gonzalez, 
C. Díez, J. N. Caamaño, B. Trigal, P. Humblot, S. Carrocera, D. Martin, E. Seli, and E. Gomez. 2014b. Metabolomic prediction of pregnancy viability in superovulated cattle embryos and recipients with Fourier transform infrared spectroscopy. Biomed. Res. Int. 2014:608579.

Peterson, A. J., and R. S. Lee. 2003. Improving successful pregnancies after embryo transfer. Theriogenology 59:687-697.

Ponsart, C., D. Le Bourhis, H. Knijn, S. Fritz, C. Guyader-Joly, T. Otter, S. Lacaze, F. Charreaux, L. Schibler, D. Dupassieux, and E. Mullaart. 2013. Reproductive technologies and genomic selection in dairy cattle. Reprod. Fertil. Dev. 26:12-21.

Reichenbach, H. D., J. Liebrich, U. Berg, and G. Brem. 1992. Pregnancy rates and births after unilateral or bilateral transfer of bovine embryos produced in vitro. J. Reprod. Fertil. 95:363-370.

Salilew-Wondim, D., M. Hölker, F. Rings, N. Ghanem, M. Ulas-Cinar, J. Peippo, E. Tholen, C. Looft, K. Schellander, and D. Tesfaye. 2010. Bovine pretransfer endometrium and embryo transcriptome fingerprints as predictors of pregnancy success after embryo transfer. Physiol. Genomics 42:201-218.

Scott, R., E. Seli, K. Miller, D. Sakkas, K. Scott, and D. H. Burns 2008. Noninvasive metabolomic profiling of human embryo culture media using Raman spectroscopy predicts embryonic reproductive potential: A prospective blinded pilot study. Fertil. Steril. 90:77-83

Seli, E., D. Sakkas, R. Scott, S. C. Kwok, S. M. Rosendahl, and D. H. Burns. 2007. Noninvasive metabolomic profiling of embryo culture media using Raman and near-infrared spectroscopy correlates with reproductive potential of embryos in women undergoing in vitro fertilization. Fertil. Steril. 88:1350-1357.

Sinclair, K. D., R. G. Lea, W. D. Rees, and L. E. Young. 2007. The developmental origins of health and disease: Current theories and epigenetic mechanisms. Soc. Reprod. Fertil. Suppl. 64:425-443.

Siqueira, L. G., C. A. Torres, E. D. Souza, P. L. Jr. Monteiro, E. K. Arashiro, L. S. Camargo, C. A. Fernandes, and J. H. Viana. 2009. Pregnancy rates and corpus luteum-related factors affecting pregnancy establishment in bovine recipients synchronized for fixedtime embryo transfer. Theriogenology 72:949-958.

Sturmey, R. G., P. Bermejo-Alvarez, A. Gutierrez-Adan, D. Rizos, H. J. Leese, and P. Lonergan. 2010. Amino acid metabolism of bovine blastocysts: A biomarker of sex and viability. Mol. Reprod. Dev. 77:285-296.

Sudano, M. J., V. G. Santos, A. Tata, C. R. Ferreira, D. M. Paschoal, R. Machado, J. Buratini, M. N. Eberlin, and F. D. Landim-
Alvarenga. 2012. Phosphatidylcholine and sphingomyelin profiles vary in Bos taurus indicus and Bos taurus taurus in vitro- and in vivo-produced blastocysts. Biol. Reprod. 87:130 http://dx.doi. org/10.1095/biolreprod.112.102897.

Sugimura, S., T. Akai, Y. Hashiyada, T. Somfai, Y. Inaba, M. Hirayama, T. Yamanouchi, H. Matsuda, S. Kobayashi, Y. Aikawa, M. Ohtake, E. Kobayashi, K. Konishi, and K. Imai. 2012. Promising system for selecting healthy in vitro-fertilized embryos in cattle. PLoS ONE 7:e36627.

Thouas, G. A., N. A. Korfiatis, A. J. French, G. M. Jones, and A. O. Trounson. 2001. Simplified technique for differential staining of inner cell mass and trophectoderm cells of mouse and bovine blastocysts. Reprod. Biomed. Online 3:25-29.

Trigal, B., E. Gómez, J. N. Caamaño, M. Muñoz, J. Moreno, S. Carrocera, D. Martín, and C. Diez. 2012. In vitro and in vivo quality of bovine embryos in vitro produced with sex-sorted sperm. Theriogenology 78:1465-1475.

Uyar, A., and E. Seli. 2012. Embryo assessment strategies and their validation for clinical use: A critical analysis of methodology. Curr. Opin. Obstet. Gynecol. 24:141-150.

Vajta, G., P. Holm, M. Kuwayama, P. J. Booth, H. Jacobsen, T. Greve, and H. Callesen. 1998. Open pulled straw (OPS) vitrification: A new way to reduce cryoinjuries of bovine ova and embryos. Mol. Reprod. Dev. 51:53-58.

Wallace, M., E. Cottell, J. Cullinane, F. M. McAuliffe, M. Wingfield, and L. Brennan. 2014. ${ }^{1} \mathrm{H}$ NMR based metabolic profiling of day 2 spent embryo media correlates with implantation potential. Syst. Biol. Reprod. Med. 60:58-63.

Witten, I. H., and E. Frank. 2005. Data Mining: Practical Machine Learning Tools and Techniques. 2nd ed. Morgan Kaufmann, San Francisco, CA.

Xu, J., Z. Guo, L. Su, T. L. Nedambale, J. Zhang, J. Schenk, J. F. Moreno, A. Dinnyés, W. Ji, X. C. Tian, X. Yang, and F. Du. 2006. Developmental potential of vitrified Holstein cattle embryos fertilized in vitro with sex-sorted sperm. J. Dairy Sci. 89:2510-2518.

Young, L. E., K. Fernandes, T. G. McEvoy, S. C. Butterwith, C. G. Gutierrez, C. Carolan, P. J. Broadbent, J. J. Robinson, I. Wilmut, and K. D. Sinclair. 2001. Epigenetic change in IGF2R is associated with fetal overgrowth after sheep embryo culture. Nat. Genet. $27: 153-154$ 